\title{
THE SECRET LIFE OF MITRAL VALVE
}

\author{
Antonio Calafiore ${ }^{1}$, Antonio Totaro ${ }^{2}$, Nicola Testa ${ }^{3}$, Cosimo Sacra $^{4}$, Gaetano Castellano ${ }^{1}$, \\ Stefano Guarracini ${ }^{5}$, Massimo Di Marco ${ }^{2}$, Sotirios Prapas ${ }^{6}$, Mario Gaudino ${ }^{7}$, Roberto \\ Lorusso $^{8}$, Domenico Paparella ${ }^{9}$, and Michele Di Mauro ${ }^{10}$ \\ ${ }^{1}$ Gemelli Molise \\ ${ }^{2}$ Affiliation not available \\ ${ }^{3}$ John Paul II Foundation for Research and Treatment \\ ${ }^{4}$ Fondazione di Ricerca e Cura Giovanni Paolo II \\ ${ }^{5}$ Private Hospital Pierangeli Srl \\ ${ }^{6}$ Henry Dunant Hospital Center \\ ${ }^{7}$ New York-Presbyterian Hospital/Weill Cornell Medical Center \\ ${ }^{8}$ Maastricht University Medical Centre \\ ${ }^{9}$ University of Bari "Aldo Moro" \\ ${ }^{10}$ Maastricht UMC+
}

September 28, 2020

\begin{abstract}
In secondary mitral regurgitation, the concept that the mitral valve (MV) is an innocent bystander, has been challenged by many studies in the last decades. The MV is a living structure with an intrinsic plasticity that reacts to changes in stretch or in mechanical stress activating bio-humoral mechanisms that have, as purpose, the adaptation of the valve to the new environment. If the adaptation is balanced, the leaflets increase both surface and length and the chordae tendinae lengthen: the result is a valve with different characteristics, but able to avoid or to limit the regurgitation. However, if the adaptation is unbalanced, the leaflets and the chords do not change their size, but become stiffer and rigid, with moderate or severe regurgitation. These changes are mediated mainly by a cytokine, the transforming growth factor $\beta$ (TGF- $\beta$ ), which is able to promote the changes that the MV needs to adapt to a new hemodynamic environment. In general, mild TGF- $\beta$ activation facilitates leaflet growth, excessive TGF- $\beta$ activation, as after a myocardial infarction, results in profibrotic changes in the leaflets, with increased thickness and stiffness. The MV is then a plastic organism, that reacts to the external stimuli, trying to maintain its physiologic integrity. This review has the goal to unveil the secret life of the MV, to understand which stimuli can trigger its plasticity and to explain why the equation "large heart=moderate/severe mitral regurgitation" and "small heart=no/mild mitral regurgitation" does not work into the clinical practice.
\end{abstract}

\section{ABSTRACT}

In secondary mitral regurgitation, the concept that the mitral valve (MV) is an innocent bystander, has been challenged by many studies in the last decades. The MV is a living structure with an intrinsic plasticity that reacts to changes in stretch or in mechanical stress activating bio-humoral mechanisms that have, as purpose, the adaptation of the valve to the new environment. If the adaptation is balanced, the leaflets increase both surface and length and the chordae tendinae lengthen: the result is a valve with different characteristics, but able to avoid or to limit the regurgitation. However, if the adaptation is unbalanced, the leaflets and the chords do not change their size, but become stiffer and rigid, with moderate or severe regurgitation. These changes are mediated mainly by a cytokine, the transforming growth factor $\beta$ (TGF- $\beta$ ), which is able 
to promote the changes that the MV needs to adapt to a new hemodynamic environment. In general, mild TGF- $\beta$ activation facilitates leaflet growth, excessive TGF- $\beta$ activation, as after a myocardial infarction, results in profibrotic changes in the leaflets, with increased thickness and stiffness. The MV is then a plastic organism, that reacts to the external stimuli, trying to maintain its physiologic integrity. This review has the goal to unveil the secret life of the MV, to understand which stimuli can trigger its plasticity and to explain why the equation "large heart=moderate/severe mitral regurgitation" and "small heart=no/mild mitral regurgitation" does not work into the clinical practice.

Keywords. Mitral valve; Mitral regurgitation; ischemic adaptation; ischemia; left ventricle.

For decades the diseases that can affect the mitral valve (MV) were considered to follow a precise scheme: infectious, inflammatory, congenital, degenerative, etc. In case of secondary mitral regurgitation (MR), the valve and its chords were supposed to remain "normal", while the regurgitation was due to geometric changes of the left ventricle (LV). If this were true, MR grade had to be directly proportional to the displacement grade of the papillary muscles (PMs). However, in the clinical setting some patients with large hearts and severely displaced PMs have sometimes absent/mild MR (fig. 1) and sometimes severe MR (fig. 2). On the other side, other patients, in spite of normal volumes and similar interpapillary distance, have either absent/mild or moderate/severe MR (fig. 3).

However, during the same time frame many studies slowly started to show that the MV was not only a passive bystander of the morphologic changes of LV geometry, but was able to induce structural modifications in response to external stimuli.

In human hearts with ischemic and non-ischemic dilated cardiomyopathy, Hueb et al. ${ }^{1}$, in a study aimed to analyze annular modifications, found that, compared with cardiomyopathy, by $24 \%$ if ischemic and by $50 \%$ if non-ischemic. The degree of ante-mortem MR was not reported. In transplant recipients, Grande-Allen et $\mathrm{al}^{2}$ demonstrated that mitral leaflets and chordae tendinae were fibrotic and showed significant changes in the extracellular matrix (ECM), with more cellularity and collagen than normal. The ALs and the PLs were longer $(+28 \%$ and $+26 \%$ respectively) and thicker $(+26 \%$ both $)$ than normal. The leaflets and the chords were biochemically different from normal and were stiffer, less extensible and less viscous than controls, due to a dysfunctional remodeling in these subset of patients ${ }^{3}$.

These studies demonstrate that the MVs and the chords of patients with end-stage dilated ischemic or nonischemic cardiomyopathy changed morphology and composition, in a process that we can term "adaptation to disease". Then the MV is not a structure that remains inert if the environment around it changes, but is able to modify its shape and its characteristics in the attempt to react to different geometry and stress distribution to avoid or to reduce regurgitation. Under this aspect the MV is a living organism, capable to respond to external stimuli due to its intrinsic plasticity (a term that defines the adaptability of a living structure to changes in its environment) in order to counteract any change that can impair its function (regurgitation). Mitral plasticity can be balanced (adaptation is complete and MR is absent or mild, as in fig. 1 and 3, A and B) or unbalanced (adaptation is uncomplete and is unable to control the MR degree, that progresses to moderate or severe, as in fig. 2 and 3, C and D).

\section{BIOLOGICAL MECHANISMS OF MV PLASTICITY}

\section{Mitral leaflets}

Mitral leaflets (MLs) consist of an extracellular matrix (ECM) layered with valvular endothelial cells (VECs), that adhere each other through a transmembrane protein (vascular endothelial (VE)-cadherin). VECs are anchored basally to the basement membrane, a 50- to 100-nm layer of specialized ECM protein complex which separates cell monolayers from the underlying connective tissue, through integrin adhesion complexes ${ }^{4}$, that mediates adhesion between the cells and their ECM. VECs can migrate into ECM changing phenotype to replenish the turnover of valvular interstitial cells (VICs), major producers of the valve ECM needed to insure durability and function. This mechanism is known as endothelial to mesenchymal transition (EndMT) (fig. 4). 
The ECM is composed of two main classes of macromolecules: proteoglycans (PGs) and fibrous proteins (collagen, elastin, fibronectin and laminin). Collagen is the most abundant fibrous protein within the interstitial ECM and constitutes up to $30 \%$ of the total protein mass of a multicellular animal. The bulk of interstitial collagen is transcribed and secreted by VICs that reside or are recruited into the ECM.

The ECM of the MV consists of four histologically distinct layers (fig.4): the atrialis and the ventricularis, both of which are thin elastin-rich layers, the spongiosa, which consists mainly of PGs, and the fibrosa, the main load-bearing layer, composed of circumferentially oriented collagen fibers. In the atrialis hemodynamic shear stresses can result in the deposition of more radially oriented collagen.

VICs, present in the ECM, are responsible for the structural integrity of the valve through protein synthesis and enzymatic degradation.

Quiescent VICs regulate low-grade ECM synthesis and degradation. Quiescent cells, in particular conditions, change phenotype and becomeactivated VICs, that take the features of myofibroblasts, facilitate valvular remodeling, but also play a role in fibrotic changes of the MV. These cells contribute to mitral adaptation, but, if the process becomes dysregulated, permanent scarring and fibrosis occurs.EndMT VICs are VECs undergoing EndMT that enter ECM and change phenotype becoming myofibroblasts. Other VICs can come from circulating blood, directly or from neovascularization, as myeloid cells that take myofibroblasts functionality.

When the integrity of the endothelial layer is reduced due to different stimuli (mechanical stress or stretching or inflammatory response following MI), cells from the circulating blood (mostly leukocytes and/or myeloid cells, which infiltrate valve tissues and differentiate into macrophages ${ }^{5}$ ) can be recruited into the valve, After having infiltrated the tissue, macrophages release inflammatory cytokines such as TNF- $\alpha$, IL- $1 \beta$, and TGF- $\beta$ leading to cardiac valve inflammation and inducing EndMT.

Mechanical stimuli that are outside of the normal physiological range induce VICs phenotypic transition into a biosynthetically active myofibroblast-like phenotype (mechanotransduction). This transition leads to increased synthesis of PGs, cytokines, as TFG- $\beta$, collagen ${ }^{6}$, as well as increased proteolytic enzyme expression and activity. This suggests that the in vivo mechanical environment plays a fundamental role in VICs response to external mechanical stimuli caused by pathological factors.

\section{Chordae Tendinae}

The chordae tendineae of the mitral valve function to transmit the contractions of the papillary muscles to the leaflets of the MV complex. They also serve to secure the leaflets to maintain valve closure and prevent mitral valve prolapse. In order to perform these functions, the chords must contain a high degree of elasticity, as well as considerable strength and endurance. These chords are composed of collagen and elastin fibers arranged in parallel. A study conducted by Millington-Sanders et al. ${ }^{7}$ showed that the chordae tendineae are composed of multiple layers of elastic fibers, an inner collagen core, and an outer layer of endothelial cells (fig. 5). During stretching, when papillary muscle contraction straightens the chords, the collagenous wavy pattern disappears and transfers the peak stress during contraction to the leaflets. The sleeve-shaped elastic network that surrounds the internal collagen suggests that on relaxation of tension, the elastic fibers would tend to restore the collagen to their wavy configuration.

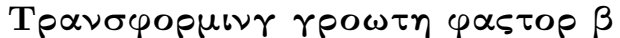

The motor of EndMT is the transforming growth factor $\beta$ (TGF- $\beta$ ) pathway, which includes multipotent cytokines that are important modulators of cell growth, inflammation, matrix synthesis and apoptosis. TGF$\beta$ regulates as well the change of VICs from quiescent to active and is able to differentiate mesenchymal cells into myofibroblasts and to regulate multiple aspects of the myofibroblast phenotype.

Beyond the TGF- $\beta$ superfamily, there are other mediators of EndMT. Several stimuli or pathways, converging with TGF- $\beta$ signaling, as shear stress ${ }^{8}$, glucose ${ }^{9}$, endothelin- ${ }^{10}$ and angiotensin $\mathrm{II}^{11}$, can stimulate EndMT (fig. 6). Others, as fibroblast growth factors and Wnt/ $\beta$-catenin signaling inhibit with different mechanisms 
EndMT. Other factors, as non-coding RNAs, can degrade the messangerRNA, inhibiting its translation into protein $^{12}$. This mechanism can favor or inhibits EndMT.

TGF- $\beta$ is secreted as a large latent complex, unable to associate with its receptors. The extracellular concentration of TGF- $\beta$ activity is primarily regulated by conversion of latent TGF- $\beta$ to active TGF- $\beta$. activation of only a small fraction of this latent TGF- $\beta$ generates maximal cellular response ${ }^{13}$. Latent TGF$\beta$ is considered to be a molecular sensor that responds to specific signals by releasing TGF- $\beta$. These signals are often associated with phenomena such as angiogenesis, wound repair, inflammation and, perhaps, cell growth. Other modes of activation have been proposed, including integrin-mediated release, which can result from mechanical force transduction ${ }^{14}$.

Activated TGF- $\beta$ mediates its effects by binding specific transmembrane receptors at the cell-membrane ${ }^{15}$ that phosphorylate specific transcription factors which translocate to the nucleus, where they regulate the transcription of specific target genes, which induce myofibroblast activation and matrix deposition. This mechanism facilitates tissue remodeling and wound healing but also plays a pathological role in fibrotic disease. After completion of remodeling activities, myofibroblasts are eliminated by apoptosis ${ }^{16}$; however, when the myofibroblast life cycle is not regulated properly, myofibroblasts persist with continued force generation and ECM production, resulting in pathological fibrosis, scarring, and fibrocontractile disease ${ }^{17}$. (fig. 7).

The renin-angiotensin system is markedly activated in response to acute myocardial infarction (AMI) and directly induces cellular responses in both cardiomyocytes and interstitial cells ${ }^{18}$. Angiotensin II stimulates fibroblast proliferation and expression of proteins ${ }^{18}$, through ECM interactions involving the Angiotensin Type 1 (AT1) receptor. Extensive evidence suggests a direct functional association between the reninangiotensin system and the TGF- $\beta$ pathway, indicating that TGF- $\beta 1$ acts downstream of Angiotensin II (fig. $6)^{18}$. In addition, angiotensin II increases VICs responsiveness to the fibrogenic actions of TGF- $\beta^{19}$.

\section{How the mitral valve increases its dimensions}

Increase of MV (leaflets and chordae tendinae) dimensions include ECM adaptation. A natural model of these changes is represented by pregnancy, where MV adapts to new hemodynamics. During pregnancy, an increased demand in oxygen to support fetal development drives an increase in maternal blood volume by up to $40 \%$. This volume overload results in cardiovascular adaptation. As the heart adapts to increased oxygen demands by ventricular hypertrophy, dilation of the periannular tissue increases the MV orifice area (around 12\%). These geometric changes alter the radius of curvature and the tension of the leaflets and are at the basis of tissue growth and remodeling ${ }^{20}$.

In pregnant bovines the AL enlarges uniformly to $40 \%$ of the basal size, in both radial and circumferential directions, with a rapid $33 \%$ increase in leaflet area within the first 2 months of pregnancy ${ }^{20}$, similar to the $35 \%$ increase in systolic leaflet area reported in an echocardiographic assessment of patients with LV dysfunction $^{21}$. Collagen fibers are crimped both in leaflet (fig. $8 \mathrm{~A}$ ) and in chordae tendinae (fig. 5). The increase of the area of the leaflets and of the length of leaflets/chordae is accompanied by a remarkable loss in collagen fiber crimp (fig. $8 \mathrm{~B}$ ), with the percentage area occupied by crimp nearly halved, and the crimp length nearly doubling. The overall thickness of the leaflets remains unchanged. The simultaneous increase in area and maintenance of thickness implies that this change is not (entirely) due to elastic deformation but also due to the addition of mass, that is, growth ${ }^{22}$. Loss of collagen crimps lengthen the leaflets, but adds mechanical compression to VICs ${ }^{23,24}$. VICs deformation (fig. 9) induces VICs phenotypic activation and subsequent transition into a biosynthetically active myofibroblast-like phenotype. Adding new fibrillar material into existing fibers, collagen crimp is gradually restored (fig. $8 \mathrm{C}$ ). VICs can be activated by compression caused by stretched collagen fibers (fig. 8 B and G), but it can be due as well to TGF- $\beta$ activation or to EndMT mediated by mechanical stress or stretching.

Based on these findings, we can speculate that MV leaflet area and leaflets/chords length increase because of loss of collagen crimp, due mainly to stretching or mechanical stress. The thickness of the leaflet/chords is maintained by addition of new collagen due to activated VICs as a consequence of mechanical forces or 
through the activation of TGF- $\beta$. New collagen is added, mainly to increase leaflets thickness. It is possible that, in a pathologic situation, variability in the intensity of the stimulus or of the cellular and humoral reaction can up or downregulate the response to the stimulus itself, and different levels of adaptation can occur. Leaflet area, leaflet/chords length can or cannot increase in proportion to the stimulus and can only thicken without increasing the size.

Then, it is possible that collagen uncrimping through mechanical stress causes mitral leaflets/chords increase of dimensions and length, and a secondary mechanism, mediated by VICs directly or stimulated by TGF$\beta$, increases the thickness of the leaflet/chords. It is likely that individual response to mechanical stress, collagen distensibility and excess of new collagen are at the basis of the different grade of mitral plasticity.

\section{Triggers that stimulate mitral valve plasticity.}

When there is a change in the dynamics of the mitral valve, induced by any cause external to the valve itself, a mechanism of adaptation starts in order to maintain the valve function.

Trigger not related to the mitral valve geometry. This is typical of aortic

regurgitation, where the trigger is the stretching of the $\mathrm{AL}$ from the regurgitatant jet ${ }^{25}$. This is able to slowly elongate the leaflet and the chords and to increase the mitral area in order to close the enlarged annular area avoiding or minimizing regurgitation (fig. 10). This mechanism of adaptation happens even if there is no MR.

Trigger related to change in mitral valve geometry. This mechanism was

demonstrated by Dal-Bianco et $\mathrm{al}^{26}$ in sheep where the PMs were retracted apically, short of producing $\mathrm{MR}$, changing only the mechanical stress exercised on the leaflets. Changing curvature is a known cause of increase of mechanical stress ${ }^{27}$ and, even without MR, it is enough to trigger a MV adaptive response. After 61 days, AL lengthened of $18 \%$ and PL of $9 \%$, with increase in thickness. Chords lengthened (anterior $20 \%$ and posterior 12\%) with increased diameter. Mechanical stresses imposed by PMs tethering increase MV leaflet area and matrix thickness, with cellular changes suggestive of reactivated embryonic valve development pathways. These findings support the concept of an actively adapting MV even in absence of regurgitation but only in presence of mechanical stress. This can happen in the initial process of non-ischemic dilated cardiomyopathy, where, in the early phase of dilation, the mitral valve is solicited to adapt to changes in stress (fig. 1).

Trigger related to mitral regurgitation. In sheep after tachycardia-induced

cardiomyopathy the presence of MR increases the mechanical stress on the valve. The mitral leaflets lengthened significantly ${ }^{28,29}$, in particular on the free edge ${ }^{29}$. Myofibroblasts were present in the extracellular matrix of as expression of change of phenotype in activated cells. In another experiment Stephens et al. ${ }^{30}$ caused MR in sheep by punching the PL and causing a hole of 2.8 to $4.8 \mathrm{~mm}$ if diameter. After 12 weeks, the AL remodeled. Elevation of matrix metalloproteinases (enzymes that are able to degrade the basal lamina allowing the cells to go through toward the matrix) mirrors an increased cellular turnover (VECs moving to the ECM). These mechanisms can follow the onset of regurgitation any cause, both anatomic and due to change of MV geometry.

Trigger related to myocardial infarction. AMI is a powerful trigger of $\mathrm{MV}$

plasticity, which often includes excessive TGF- $\beta$ upregulation. This can be due to post-MI angiotensin II activation, which in turn can activate TGF- $\beta$ expression enhancing its profibrotic activity ${ }^{6}$.

Since 1997, it was noted in an experimental setting by Quick et al. ${ }^{31}$ that induction of AMI with/out mitral regurgitation caused upregulation of collagen in the MV, mainly in the AL. In a consecutive series of 91 patients with inferior $\mathrm{AMI}^{32}, 53.7 \%$ developed MR moderate or more, $28.4 \%$ in patients who showed mitral plasticity $(60 / 91,66 \%)$ and $67.8 \%$ in patients who did not $(31 / 91,34 \%)(\mathrm{p}<0.003)$. Interestingly, the most striking differences in length in patients with no or mild MR vs. moderate or more MR were seen for the AL 
(25.5 \pm 3.3 vs $22.4 \pm 3.1 \mathrm{~mm} ; \mathrm{p}<0.001)$ and the chordal length $(28.4 \pm 7.2$ vs. $24.1 \pm 8.2 \mathrm{~mm} ; \mathrm{p}<0.01)$. The PL length difference was not significant $(16.1 \pm 2.6$ vs. $15 \pm 3.1 \mathrm{~mm} ; \mathrm{p}=0.08)$. It is as well evident in many studies that the $\mathrm{AL}$ increases its length more than the $\mathrm{PL}^{32,33}$.

In patients who had AMI, and early revascularization in almost all cases, Beaudoin et $\mathrm{al}^{34}$ demonstrated increased thickness of the mitral leaflets 2 years post AMI and a further increase thereafter. There was a progressive reduction of the AL excursion and patients who developed more than mild MR had thicker mitral leaflets than patients who did not.

It is worth noting the importance of the role of chordae tendinae in the process of mitral plasticity. The length of the chords is essential to reduce the regurgitation grade. In patients with ischemic and non-ischemic cardiomyopathy chords longer $10 \mathrm{~mm}$ than normal contributed, in large hearts, to reduce MR grade to absent or mild ${ }^{33}$. In patients with the same annular diameter and MV leaflet area, a difference of $2.5 \mathrm{~mm}$ in chordal length made the difference from MR grade moderate or more and mild or absent, having this latter group larger LV volumes ${ }^{35}$.

\section{CONCLUSIONS}

Stretching and mechanical stress are the initial mechanisms that trigger MV adaptation to a new hemodynamic situation. Increase in leaflets area and length and in chordal length is due to collagen uncrimping, which is able to increase leaflets and chordal dimensions by 30 to $50 \%$. To maintain the same thickness, it is necessary to produce more collagen by activated VICs, either because of mechanical stress or TGF- $\beta$ activation. The purpose is to maintain the same thickness even with a longer or larger MV, but the process can become dysregulated, such as in presence of an AMI.

TGF- $\beta$ is present in the MV in a latent form. Many stimuli (stretching, mechanical stress, inflammation, turbulent flow, and others), can trigger the release of active TGF- $\beta$, which binds to a membrane receptor that in turn activate signal transducers that accumulate into the nuclei and transcripts specific genes, targeted to induce fibrotic changes. Targets can be quiescent VICs, present in the ECM, that change in activated myofibroblasts, but VECs as well. As a consequence, VECs activate and lose the adhesion to neighbor cells. The basement membrane is eroded by enzymatic secretion and the VECs migrate into the ECM, where transition to mesenchymal cells, which in turn can differentiate in other cells, such as myofibroblasts, etc. Myofibroblasts produce collagen and the excess of collagen production causes an excessive accumulation of ECM components. If this process becomes dysregulated, as often it happens after AMI, permanent scarring and increased leaflet fibrosis occurs. After a MI, VECs undergo EndMT, VICs are activated to become myofibroblasts that secrete and compact ECM, and there is evidence for valve neovascularization and leukocyte infiltration ${ }^{6,26,36}$. Infiltrating macrophages and leukocytes release growth factors and cytokines, such as TGF- $\beta$ family members, which can further promote angiogenesis, collagen production, attraction of additional inflammatory cells, and transform VICs to myofibroblasts that also secrete growth factors and cytokines.

Activated TGF- $\beta$ can induce phenotype changes in quiescent VICs, which change morphology and function, contributing to pathologic changes. In the ischemic setting, excessive TGF- $\beta$ signaling occurs, that stimulates exuberant EndMT, resulting in profibrotic changes of the leaflets, such as markedly increased thickness, cellular proliferation, and excessive ECM turnover with collagen deposition.

It is not clear if the activation of the ECs is directly dependent by the trigger, such as mechanical stress, or induced by TGF- $\beta$ since the beginning.

\section{Therapeutic implications}

The different anatomical pictures that result from MV plasticity can provide many anatomical aspects. The most favorable is a balanced adaptation, with long leaflets and long chords, with absent or mild MR. On the other extreme there is an unbalanced adaptation, where leaflet area and length are not adequate to respond to increased annular area and changed LV geometry. Leaflets are fibrotic and chords are thick and retracted, with resulting moderate or severe MR. In the middle there are many possibilities, with different 
anatomical aspects and MR grade. Among them, it is worthy noting that we can have partial adaptation , such as long leaflets and tethered chords or relatively short and thick leaflets and normal or long chords. Then the necessity to look carefully at the anatomy of the MV apparatus, which can change from case to case.

The comprehension, even if partial, of the mechanisms of adaptation of the MV led to the search of pharmacological prevention of a possible maladaptive evolution of the process. Given the role of angiotensin II as TGF- $\beta$ activator after MI, the use of losartan, a selective inhibitor of angiotensin II receptor-1, was explored successfully both in vitro ${ }^{37}$ and in an animal model ${ }^{38}$. In this latter experiment profibrotic changes of tethered MV leaflets post-MI were modulated by losartan without eliminating adaptive growth. Losartan decreases production of TGF- $\beta$ and its receptor and angiotensin II-induced release of latent TGF- $\beta^{39}$. It blocks the interaction of angiotensin II with its AT1 receptor, decreasing TGF- $\beta$ signaling ${ }^{18}$. Losartan inhibits angiotensin II-induced expression of endoglin, which promotes the fibrogenic effects of TGF- $\beta^{19}$. Through such effects on the MV, losartan can potentially inhibit fibrosis ${ }^{40}$, while maintaining adaptive leaflet growth with flexible leaflet closure.

In a clinical study on 40 patients with 2 serial echocardiograms (6 days and 12 years) after an inferior MI, leaflet thickness increased over time and was correlated with MR. Most of the patients were treated with angiotensin converting enzyme inhibitors or angiotensin receptor blockers. In the subgroup taking high dose, late echocardiogram showed thinner leaflets than in patients where small dose was used ${ }^{34}$. In another study on patients with end stage renal disease, losartan more effectively suppressed myocardial fibrosis than did enalapril or amlodipine despite a comparable antihypertensive effect among the three drugs ${ }^{41}$.

The length of the leaflets can be used as a predictor of the reversibility of untreated mild or moderate IMR after CABG . Yoshida et al. ${ }^{42}$ found that the estimated coaptation length was a determinant of MR improvement after 2.9 years from surgery, with a cutoff of $6.5 \mathrm{~mm}$. In patients where MR improved, leaflets were longer than in patients where MR did not improve.

Finally, understanding the mechanisms of mitral plasticity can provide a new paradigm in surgical treatment of IMR. Surgical repair with reductive mitral annuloplasty alone imposes abnormal biomechanics on the MV. Serial cardiac computed tomography 1 to 5 years postoperatively demonstrated that MV leaflet thickening occurred in $69 \%$ of 45 patients $^{43}$. Patients with thicker leaflets also presented with elevated transmitral pressure gradients, indicating progressive valve stenosis. In a swine experimental model of moderate IMR pathogenic biological changes in the MV leaflets were demonstrated within a short period of 3 months. Collagen levels were elevated in these stressed leaflets and the profibrotic changes in the valve paralleled increase in TGF- $\beta^{44}$. Surgical repair with restrictive mitral annuloplasty alone frequently do not restore physiological leaflet configuration and, if mechanical strains remains elevated, pathogenic changes in valve leaflets can be induced.

The role of reparative surgery should be to "take up where the nature left" and complete the process of adaption by intervening at different levels on the MV and mimicking the natural adaptive mechanism. We have termed this surgical approach "surgical mitral plasticity" 45 . To complete what nature could not, the AL has to be augmented and lengthened (by an autologous glutaraldehyde-treated or heterologous pericardial patch or any biological patch) and the second order chords have to be cut to increase the effective AL length and area (fig. 11). It is worth emphasizing the necessity of second-order chords transection, as the fibrotic process, once started, can be progressive ${ }^{46}$. Moreover, by eliminating apical tethering, the AL can recover its normal curvature by removal, or reduction, of the tenting area. In selected cases, when the AL is sufficiently long, it is not necessary to augment it, but chordal cutting, performed through aortotomy, is sufficient (fig. 12). This maneuver causes an important reduction of leaflets stress ${ }^{27}$. Restrictive mitral annuloplasty should then complete the repair. None of these technique is new, but the rationale behind is. We prefer to target the surgical augmentation on the AL as the PL, after restrictive mitral annuloplasty, is positioned vertically, representing a doorjamb for the AL which, as recovers its length and mobility, is able to close the annular area with a long coaptation length. 
Mechanisms of failure after isolated annuloplasty can be due to shortness and thickness of the AL, with persistent chordal tethering and critical tenting area (fig. 13). Many techniques to be added to isolated restrictive mitralo annuloplasty have been described and are currently used to improve results of MV repair. A longer follow up and a critical review of the results will give us a guide to correct secondary MR, a disease more complex than perceived.

\section{FIGURES}

Fig. 1 - Patients with idiopathic dilated cardiomyopathy, severely dilated hearts, displaced papillary muscles and mild MR. Balanced mitral adaptation, with longer leaflets and chords.

Legend. EDV, end diastolic volume; EDD, end diastolic dimension; EF, ejection fraction; IPd, interpapillary distance; AML, anterior mitral leaflet; PML, posterior mitral leaflet; MR, mitral regurgitation.

Fig. 2 - Patient with idiopathic dilated cardiomyopathy, severely dilated heart, dispiace papillary muscles and severe MR. Unbalanced mitral adaptation, with nor elongated leaflets and tethered chords.

Legend. EDV, end diastolic volume; EDD, end diastolic dimension; EF, ejection fraction; IPd, interpapillary distance; AML, anterior mitral leaflet; PML, posterior mitral leaflet; MR, mitral regurgitation.

Fig. 3 - Patients with ischemic cardiomyopathy after an anterior AMI, not dilated heart and distance between the papillary muscles within the range. A and B, mild MR: C and D, severe MR.

Legend. AMI, acute myocardial infarction; EDV, end diastolic volume; EDD, end diastolic dimension; EF, ejection fraction; AML, anterior mitral leaflet; PML, posterior mitral leaflet; MR, mitral regurgitation.

Fig. 4 - The leaflet consists of four layers, the atrialis and the ventricularis, both of which are thin elastin-rich layers, the spongiosa, which consists mainly of GAGs and collagen, and the fibrosa, the main load-bearing layer, composed of circumferentially oriented collagen fibers. In a stressed valve, or in a valve stimulated by TGF- $\beta$, the endothelial cells undergo EndMT, increasing the number of matrix-producing cells.

Legend. GAGs, glycosaminoglycans; TGF- $\beta$ - transforming growth factor $\beta$. EndMT, endothelial-tomesenchimal transition; VIC, valve interstitial cell.

From Levine et $\mathrm{al}^{47}$, with permission.

Fig. 5 - Scanning electron micrograph of external aspect of the endothelial cells of the chorda, obtained from a 23-y-old subject (x3170). (B ) The elastic fibers, situated underneath the endocardium which was removed (x1720). ( $C$ ) Interior of a split chorda. Waves of collagen fibrils with similar dimensions $(10.7 \mu \mathrm{m})$ to the reflections shown in $A$ and undulations in $B$ (x3260).

From Millington-Sanders and Stolinski ${ }^{7}$, with permission.

Fig. 6 - Diagrammatic representation of other pathways involved in EndMT regulation. Molecular signaling pathways beside the TGF- $\beta$ pathways that induce or inhibit the EndMT process. These include endothelin (ET)-1, NOTCH, caveolin (CAV)-1, Wnt, high glucose, and hypoxia inducible factor (HIF)-1 $\alpha$ pathways. Shear stress forces (represented by undulating arrows) induce EndMT through several different molecular mechanisms. One mechanism is initiated by the mechanical force-induced release and liberation of TGF- $\beta$ from the latency associated peptide (LAP) followed by activation of the TGF- $\beta$ canonical pathway. Legend. EndMT, endothelial-to-mesenchymal transcription; TGF- $\beta$, transforming growth factor- $\beta$.

From Piera-Velazquez and Jimenez ${ }^{48}$, with permission.

Fig. 7 - Myofibroblasts play a central role in in the progression of fibrotic disease in the heart. One important mechanism yielding MyoFBs is EndMT (A), by which endothelial cells lose their endothelial markers. Valvular interstitial cells and cardiac fibroblasts can differentiate into MyoFBs in response to high mechanical strain (B) with the degradation of initial ECM and a corresponding decrease in tissue stiffness. Quiescent VICs and CFs can also differentiate into MyoFBs in response to high mechanical stress (C), caused by both increased tissue stiffness and increased tissue forces. MyoFBs increase the overall stress in the 
environment by producing excess ECM and contracting existing ECM through increased cellular contractility. MyoFBs also release profibrotic signaling factors, including TGF- $\beta 1$ and Wnt, that promote further MyoFB differentiation and tissue stiffening. This forms a positive feedback loop leading to progressively worsening fibrosis.

Legend. MyoFBs, myofibroblasts; ECM, exracellular matrix; VICs, valve interstitial cells; CFs, cardiac fibroblasts; TGF- $\beta 1$, transforming growth factor- $\beta 1$; Wnt, wingless Int-1; EC, endothelial cell; EndMT, endothelial-to-mesenchimal transition; FGF, fibroblast growth factor; $\alpha$-SMA, smooth muscle $\alpha$-actin.

From Schroer and Merryman ${ }^{49}$, with permission.

Fig. 8 - A, anterior leaflet of a never-pregnant heifer. B , anterior leaflet of a cow in early phase of pregnancy (110 days). C, anterior leaflet of a cow in the late phase of pregnancy (240 days). Collagen fibers are crimped in heifers, but lose crimp in early pregnancy. Cells are notably deformed, possibly becoming activated VICs. In late pregnancy crimping is restored, but the period is almost the double than in heifer. $\mathrm{D}$, schematic of the possible mechanism of valvular growth via the serial addition of collagenous fibrillar material as well as the deposition of new collagen fibres. As existing fibres elongate, their crimp amplitude and slack are gradually restored, relieving VICs compression and restoring normal homeostatic function.

Legend. VICs. Valvular interstitial cells.

From Rego et $\mathrm{al}^{23}$, with permission

Fig. 9 - Cellular deformation demonstrate nonlinear tissue micromechanics at higher strain levels. Mitral valve interstitial cells from the fibrosa layer from samples stretched at different strain levels (increasing from left to right). Scale bar: $5 \mu \mathrm{m}$.

Legend. T, transmural/thickness; C, circumferential; NOI, normalized orientation index. A NOI value of $100 \%$ represents a highly oriented fibre network, whereas a NOI value of $0 \%$ represents a more randomly oriented network.

From Ayoub et $\mathrm{al}^{24}$, with permission.

Fig. 10 - Patients with dilated cardiomyopathy due to severe aortic regurgitation. The heart is severely dilated heart, but MR is mild. Balanced mitral adaptation, with longer leaflets and chords.

Legend. EDV, end diastolic volume; EDD, end diastolic dimension; EF, ejection fraction; IPd, interpapillary distance; AML, anterior mitral leaflet; PML, posterior mitral leaflet; MR, mitral regurgitation.

Fig. $11-3 \mathrm{D}$ reconstruction of the mitral annulus and leaflets in systole. Severe chronic IMR. A, preoperatively the tenting volume is high and the AL is directed toward the apex because of tethering of the second-order chords. B, postoperatively the tenting volume is minimal and the AL reaches easily the PL because of the increased length and the lack of tethering. The PL is positioned vertically.

Legend. 3D, three-dimensional; AL, anterior leaflet; IMR, ischemic mitral regurgitation; PL, posterior leaflet

From Calafiore et $\mathrm{al}^{45}$, with permission.

Fig. 12 - Patient with severe dilated cardiomyopathy. A, severe MR, with a long AL and tethering of the second order chords (B, red arrow). 3D reconstruction of mitral annulus and leaflets in systole. $\mathrm{C}$, the AL is moved toward the apex and the second order chords are tethered (red arrow). D, after mitral annuloplasty and second order cutting through aortotomy, the AL coapts with the PL with a coaptation length of $9 \mathrm{~mm}$. Chordal tethering disappeared.

Legend. MR, mitral regurgitation; AL, anterior leaflet; PL, posterior leaflet.

Fig. 13 - Failure after isolated restrictive mitral annuloplasty for ischaemic mitral regurgitation. (A) Transthoracic echocardiography. The anterior leaflet is short $(21 \mathrm{~mm})$ and tethered (red arrow) and is not able to coapt with the posterior leaflet. There is moderate mitral regurgitation. (B and C) Transoesophageal 
echocardiography. There is a significant tenting volume, which pushes the mitral valve inside the left ventricle. The anterior leaflet has reduced mobility and cannot coapt with the posterior leaflet due to chordal tethering.

From Calafiore et $\mathrm{al}^{50}$, with permission.

\section{REFERENCES}

1. Hueb AC, Jatene FB, Moreira LF, Pomerantzeff PM, Kallas E, de Oliveira SA. Ventricular remodeling and mitral valve modifications in dilated cardiomyopathy: new insights from anatomic study. J Thorac Cardiovasc Surg 2002;124:1216-24.

2. Grande-Allen KJ, Borowski AG, Troughton RW, et al. Apparently normal mitral valves in patients with heart failure demonstrate biochemical and structural derangements: an extracellular matrix and echocardiographic study. J Am Coll Cardiol 2005;45:54-61.

3. Grande-Allen KJ, Barber JE, Klatka KM, et al. Mitral valve stiffening in end-stage heart failure: evidence of an organic contribution to functional mitral regurgitation. J Thorac Cardiovasc Surg 2005;130:783-90.

4. Hynes RO. Integrins: bidirectional, allosteric signaling machines. Cell 2002;110:673-87.

5. Aikawa E, Nahrendorf M, Sosnovik D, et al. Multimodality molecular imaging identifies proteolytic and osteogenic activities in early aortic valve disease. Circulation 2007;115:377-86.

6. Dal-Bianco JP, Aikawa E, Bischoff J, et al. Myocardial Infarction Alters Adaptation of the Tethered Mitral Valve. J Am Coll Cardiol 2016;67:275-87.

7. Millington-Sanders C, Meir A, Lawrence L, Stolinski C. Structure of chordae tendineae in the left ventricle of the human heart. J Anat 1998;192 ( Pt 4):573-81.

8. Mahmoud MM, Serbanovic-Canic J, Feng S, et al. Shear stress induces endothelial-to-mesenchymal transition via the transcription factor Snail. Sci Rep 2017;7:3375.

9. Yu CH, Suriguga, Gong M, et al. High glucose induced endothelial to mesenchymal transition in human umbilical vein endothelial cell. Exp Mol Pathol 2017;102:377-83.

10. Widyantoro B, Emoto N, Nakayama K, et al. Endothelial cell-derived endothelin-1 promotes cardiac fibrosis in diabetic hearts through stimulation of endothelial-to-mesenchymal transition. Circulation 2010;121:2407-18.

11. Tang RN, Lv LL, Zhang JD, et al. Effects of angiotensin II receptor blocker on myocardial endothelialto-mesenchymal transition in diabetic rats. Int J Cardiol 2013;162:92-9.

12. Gu S, Kay MA. How do miRNAs mediate translational repression? Silence 2010;1:11.

13. Annes JP, Munger JS, Rifkin DB. Making sense of latent TGFbeta activation. J Cell Sci 2003;116:217-24.

14. Robertson IB, Horiguchi M, Zilberberg L, Dabovic B, Hadjiolova K, Rifkin DB. Latent TGF-beta-binding proteins. Matrix Biol 2015;47:44-53.

15. Feng XH, Derynck R. Specificity and versatility in tgf-beta signaling through Smads. Annu Rev Cell Dev Biol 2005;21:659-93.

16. Desmouliere A, Redard M, Darby I, Gabbiani G. Apoptosis mediates the decrease in cellularity during the transition between granulation tissue and scar. Am J Pathol 1995;146:56-66.

17. Desmouliere A, Gabbiani G. The role of the myofibroblast in wound healing and fibrocontractive diseases. In: Clark RAF, ed. The Molecular and Cellular Biology of Wound Repair. New York, NY: Plenum Press; 1996

18. Rosenkranz S. TGF-beta1 and angiotensin networking in cardiac remodeling. Cardiovasc Res 2004;63:423-32. 
19. Chen K, Mehta JL, Li D, Joseph L, Joseph J. Transforming growth factor beta receptor endoglin is expressed in cardiac fibroblasts and modulates profibrogenic actions of angiotensin II. Circ Res 2004;95:116773.

20. Pierlot CM, Lee JM, Amini R, Sacks MS, Wells SM. Pregnancy-induced remodeling of collagen architecture and content in the mitral valve. Ann Biomed Eng 2014;42:2058-71.

21. Chaput M, Handschumacher MD, Tournoux F, et al. Mitral leaflet adaptation to ventricular remodeling: occurrence and adequacy in patients with functional mitral regurgitation. Circulation 2008;118:845-52.

22. Pierlot CM, Moeller AD, Lee JM, Wells SM. Pregnancy-induced remodeling of heart valves. Am J Physiol Heart Circ Physiol 2015;309:H1565-78.

23. Rego BV, Wells SM, Lee CH, Sacks MS. Mitral valve leaflet remodelling during pregnancy: insights into cell-mediated recovery of tissue homeostasis. J R Soc Interface 2016;13.

24. Ayoub S, Lee CH, Driesbaugh KH, et al. Regulation of valve interstitial cell homeostasis by mechanical deformation: implications for heart valve disease and surgical repair. J R Soc Interface 2017;14.

25. Beaudoin J, Handschumacher MD, Zeng X, et al. Mitral valve enlargement in chronic aortic regurgitation as a compensatory mechanism to prevent functional mitral regurgitation in the dilated left ventricle. J Am Coll Cardiol 2013;61:1809-16.

26. Dal-Bianco JP, Aikawa E, Bischoff J, et al. Active adaptation of the tethered mitral valve: insights into a compensatory mechanism for functional mitral regurgitation. Circulation 2009;120:334-42.

27. Salgo IS, Gorman JH, 3rd, Gorman RC, et al. Effect of annular shape on leaflet curvature in reducing mitral leaflet stress. Circulation 2002;106:711-7.

28. Stephens EH, Timek TA, Daughters GT, et al. Significant changes in mitral valve leaflet matrix composition and turnover with tachycardia-induced cardiomyopathy. Circulation 2009;120:S112-9.

29. Timek TA, Lai DT, Dagum P, et al. Mitral leaflet remodeling in dilated cardiomyopathy. Circulation 2006;114:I518-23.

30. Stephens EH, Nguyen TC, Itoh A, Ingels NB, Jr., Miller DC, Grande-Allen KJ. The effects of mitral regurgitation alone are sufficient for leaflet remodeling. Circulation 2008;118:S243-9.

31. Quick DW, Kunzelman KS, Kneebone JM, Cochran RP. Collagen synthesis is upregulated in mitral valves subjected to altered stress. ASAIO J 1997;43:181-6.

32. Avila-Vanzzini N, Michelena HI, Fritche Salazar JF, et al. Clinical and echocardiographic factors associated with mitral plasticity in patients with chronic inferior myocardial infarction. Eur Heart J Cardiovasc Imaging 2018;19:508-15.

33. Yoshida S, Fukushima S, Miyagawa S, et al. The Adaptive Remodeling of the Anterior Mitral Leaflet and Chordae Tendineae Is Associated with Mitral Valve Function in Advanced Ischemic and Nonischemic Dilated Cardiomyopathy. Int Heart J 2018;59:959-67.

34. Beaudoin J, Dal-Bianco JP, Aikawa E, et al. Mitral Leaflet Changes Following Myocardial Infarction: Clinical Evidence for Maladaptive Valvular Remodeling. Circ Cardiovasc Imaging 2017;10.

35. Obase K, Weinert L, Hollatz A, et al. Elongation of chordae tendineae as an adaptive process to reduce mitral regurgitation in functional mitral regurgitation. Eur Heart J Cardiovasc Imaging 2016;17:500-9.

36. Walker GA, Masters KS, Shah DN, Anseth KS, Leinwand LA. Valvular myofibroblast activation by transforming growth factor-beta: implications for pathological extracellular matrix remodeling in heart valve disease. Circ Res 2004;95:253-60. 
37. Wylie-Sears J, Levine RA, Bischoff J. Losartan inhibits endothelial-to-mesenchymal transformation in mitral valve endothelial cells by blocking transforming growth factor-beta-induced phosphorylation of ERK. Biochem Biophys Res Commun 2014;446:870-5.

38. Bartko PE, Dal-Bianco JP, Guerrero JL, et al. Effect of Losartan on Mitral Valve Changes After Myocardial Infarction. J Am Coll Cardiol 2017;70:1232-44.

39. Peng H, Carretero OA, Vuljaj N, et al. Angiotensin-converting enzyme inhibitors: a new mechanism of action. Circulation 2005;112:2436-45.

40. Leask A. Potential therapeutic targets for cardiac fibrosis: TGFbeta, angiotensin, endothelin, CCN2, and PDGF, partners in fibroblast activation. Circ Res 2010;106:1675-80.

41. Shibasaki Y, Nishiue T, Masaki H, et al. Impact of the angiotensin II receptor antagonist, losartan, on myocardial fibrosis in patients with end-stage renal disease: assessment by ultrasonic integrated backscatter and biochemical markers. Hypertens Res 2005;28:787-95.

42. Yoshida S, Fukushima S, Miyagawa S, et al. Mitral Valve Structure in Addition to Myocardial Viability Determines the Outcome of Functional Mitral Regurgitation After Coronary Artery Bypass Grafting. Circ J 2017;81:1620-7.

43. Suh YJ, Chang BC, Im DJ, et al. Assessment of mitral annuloplasty ring by cardiac computed tomography: Correlation with echocardiographic parameters and comparison between two different ring types. J Thorac Cardiovasc Surg 2015;150:1082-90.

44. Sielicka A, Sarin EL, Shi W, et al. Pathological Remodeling of Mitral Valve Leaflets from Unphysiologic Leaflet Mechanics after Undersized Mitral Annuloplasty to Repair Ischemic Mitral Regurgitation. J Am Heart Assoc 2018;7:e009777.

45. Calafiore AM, Totaro A, De Amicis V, et al. Surgical mitral plasticity for chronic ischemic mitral regurgitation. J Card Surg 2020.

46. Calafiore AM, Totaro A, Testa N, Sacra C, Calvo E, Di Mauro M. Association of tethering of the second-order chords and prolapse of the first-order chords of the anterior leaflet: A risk factor for early and late repair failure. J Card Surg 2020.

47. Levine RA, Hagege AA, Judge DP, et al. Mitral valve disease-morphology and mechanisms. Nat Rev Cardiol 2015;12:689-710.

48. Piera-Velazquez S, Jimenez SA. Endothelial to Mesenchymal Transition: Role in Physiology and in the Pathogenesis of Human Diseases. Physiol Rev 2019;99:1281-324.

49. Schroer AK, Merryman WD. Mechanobiology of myofibroblast adhesion in fibrotic cardiac disease. J Cell Sci 2015;128:1865-75.

50. Calafiore AM, Totaro A, Paparella D, et al. Mimicking natural mitral adaptation to ischaemic regurgitation: a proposed change in the surgical paradigm. Eur J Cardiothorac Surg 2020;58:35-9. 

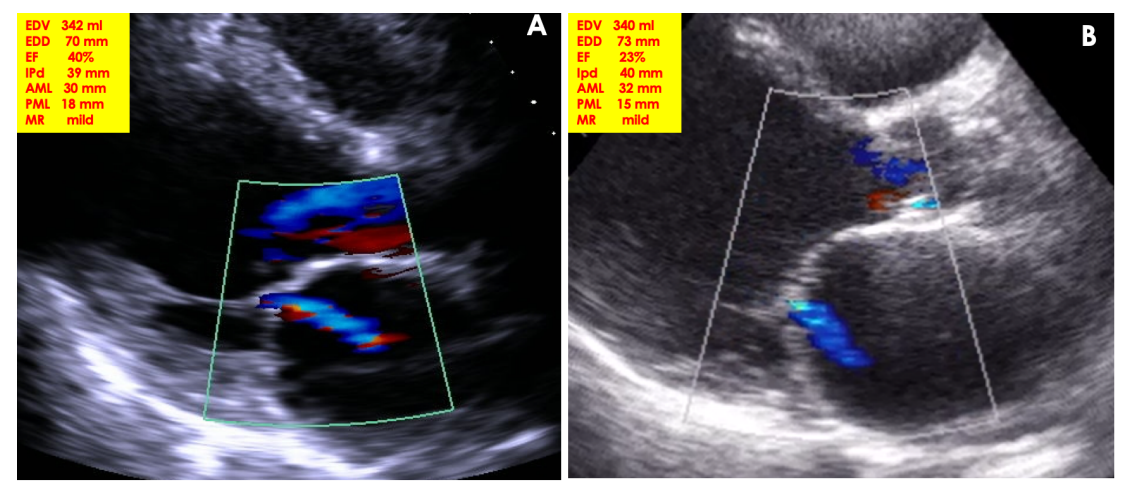

Fig. 1
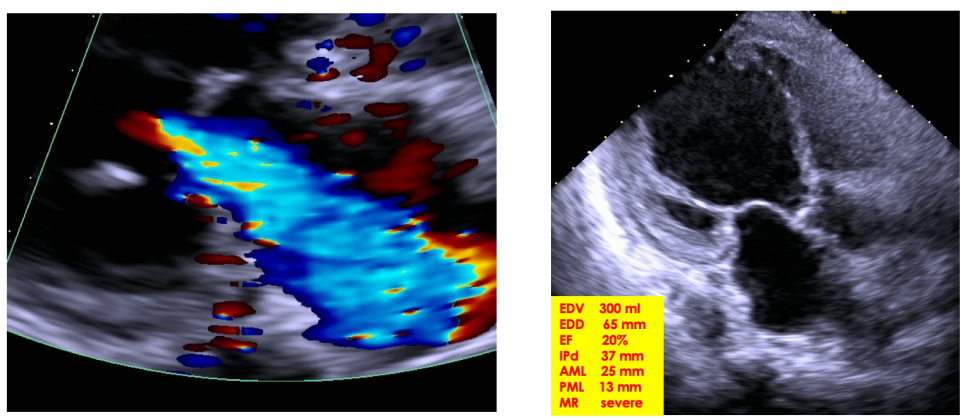

Fig. 2
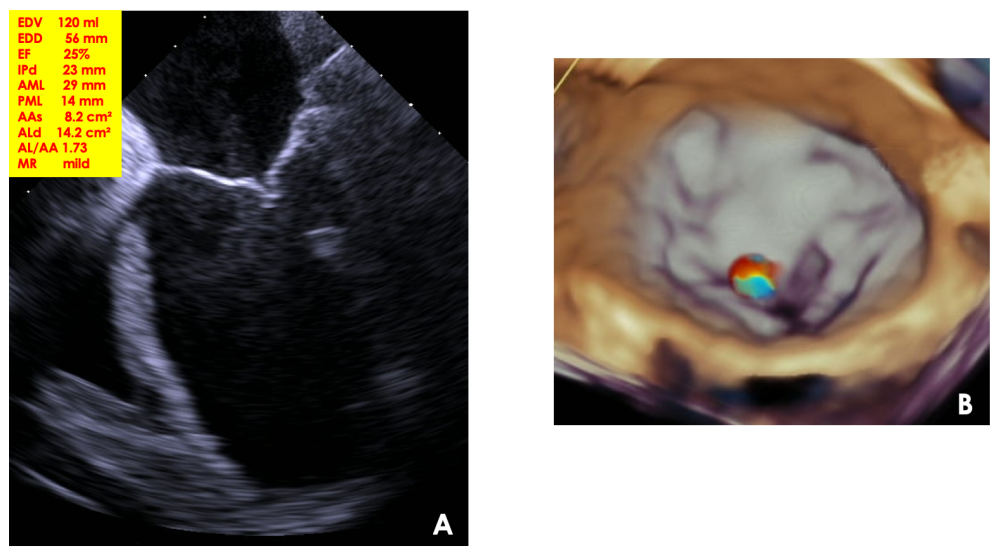

Fig. 3, A and B 

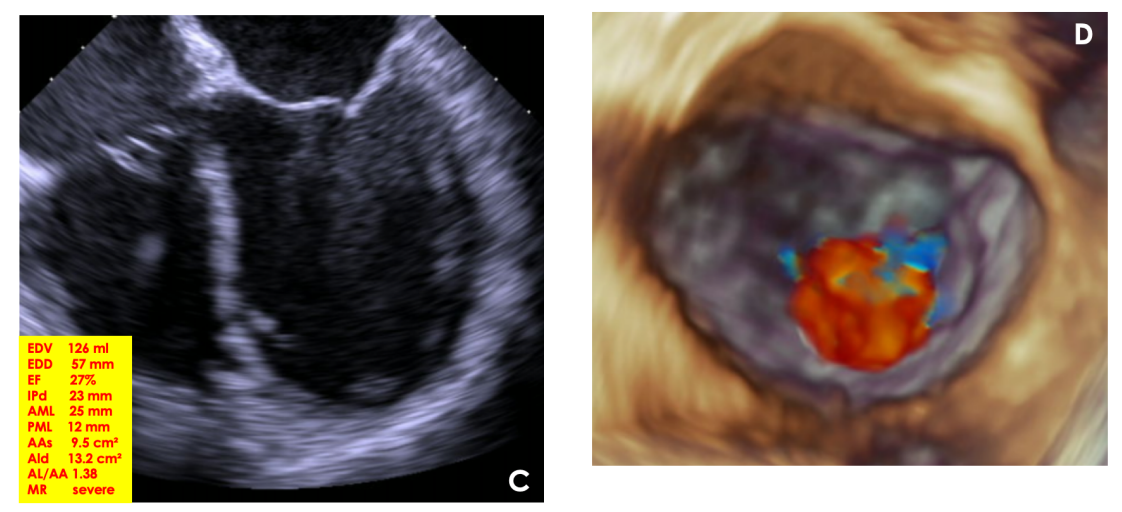

Fig. 3, C and D

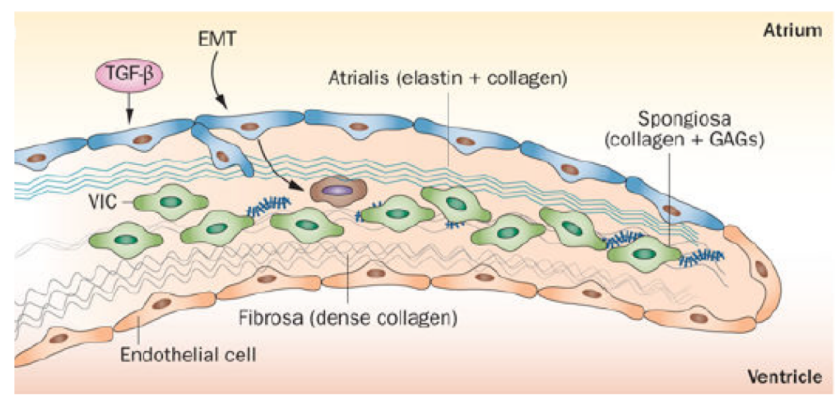

fig. 4
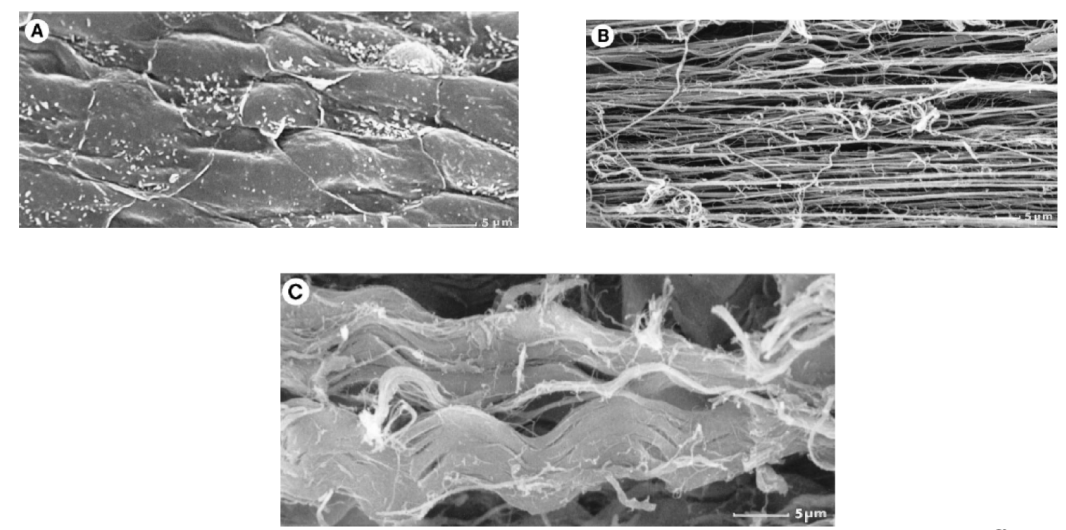

fig. 5 


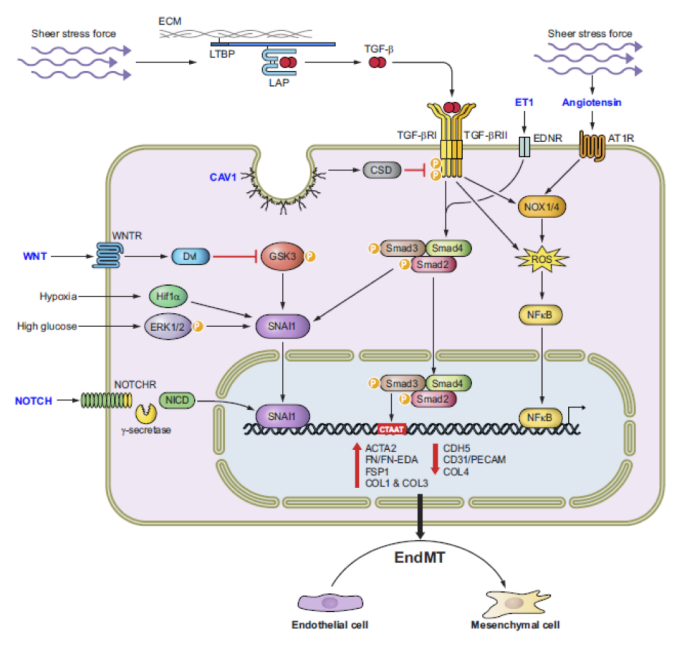

fig. 6

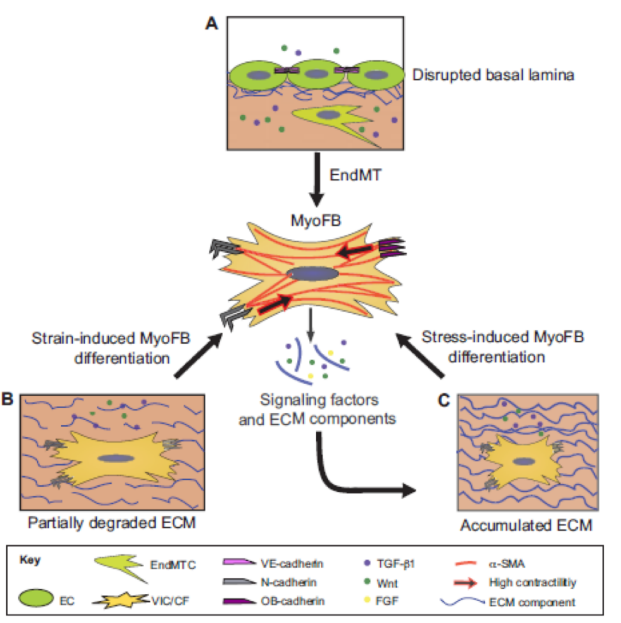

fig. 7
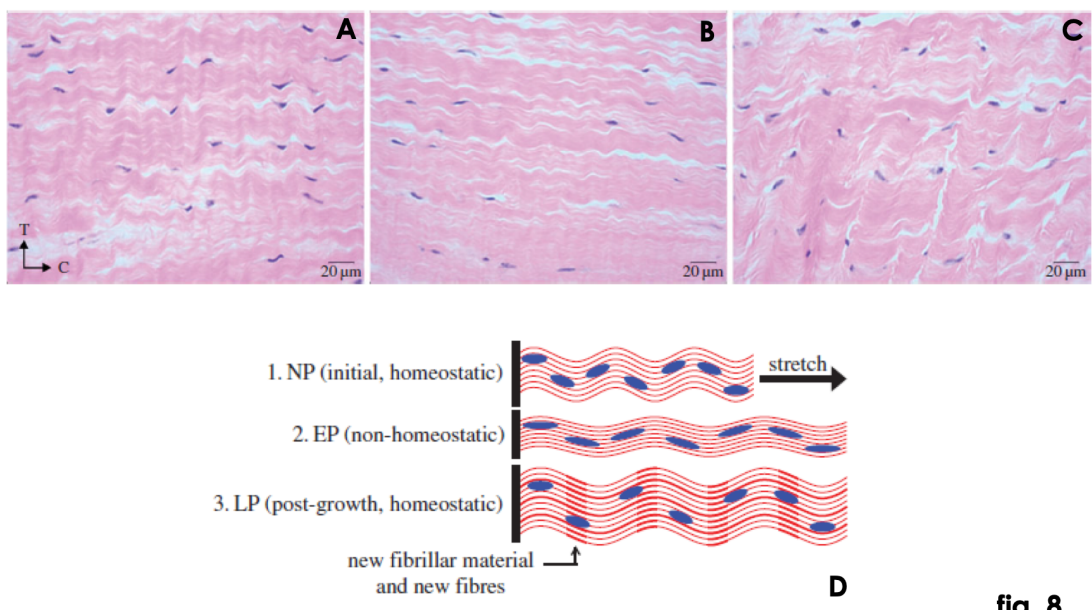

fig. 8 


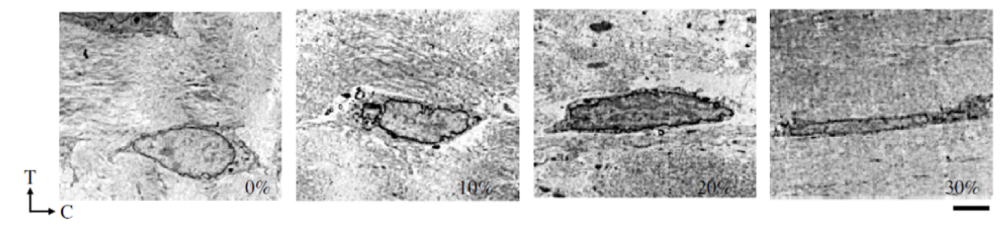

fig. 9
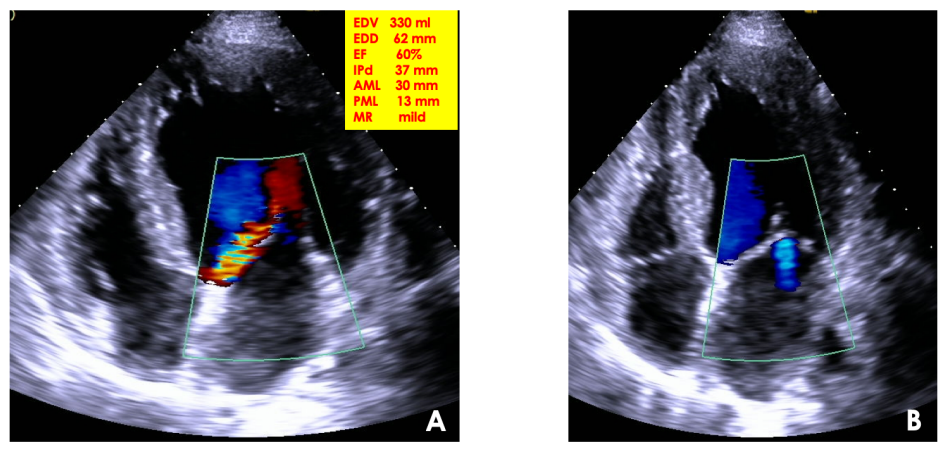

Fig. 10
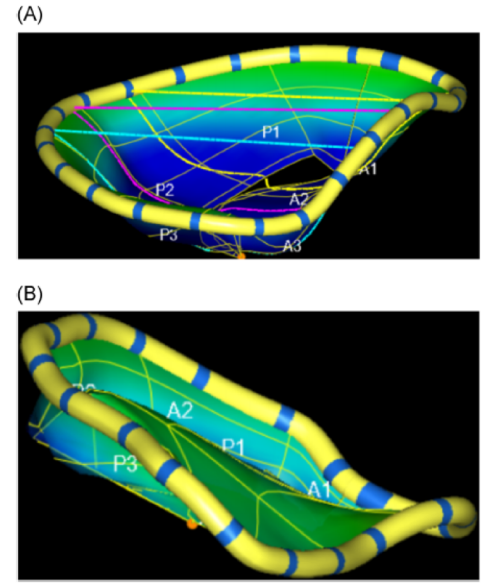

Fig. 11 

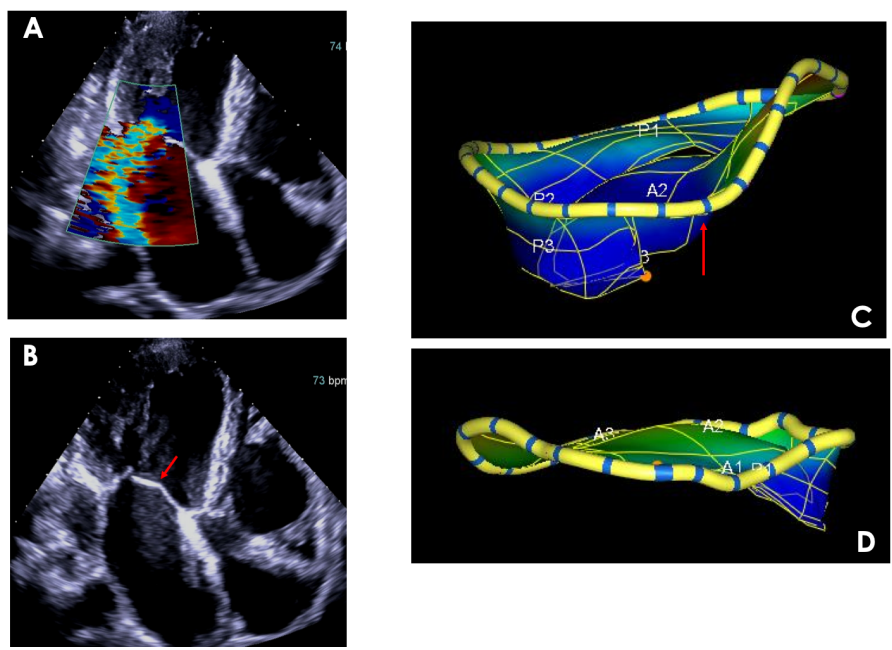

Fig. 12
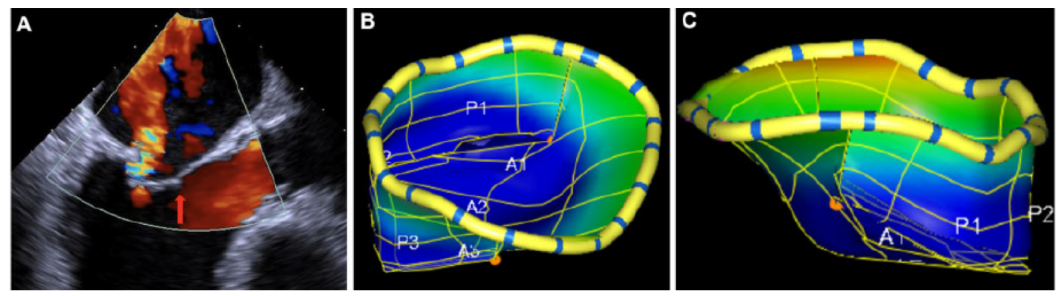

Fig. 13 